\title{
Research Paper \\ Investigation on the Efficiency of Modified Fenton on the Treatment of Leachate Generated From Oily Sludge Composting
}

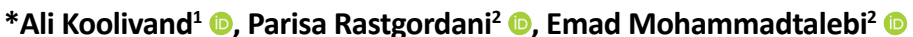

1. Department of Environmental Health Engineering, Faculty of Health, Arak University of Medical Sciences, Arak, Iran.

2. Student Research Committee, Arak University of Medical Sciences, Arak, Iran.

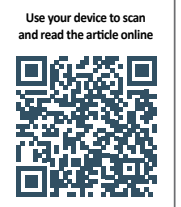

Citation: Koolivand A, Rastgordani P, Mohammadtalebi E. [Investigation on the Efficiency of Modified Fenton on the Treatment of Leachate Generated From Oily Sludge Composting (Persian)]. Journal of Arak University of Medical Sciences (JAMS). 2021; 24(3):324-333. https://doi.org/10.32598/JAMS.24.3.2117.8

doi https://doi.org/10.32598/JAMS.24.3.2117.8

Key words:

Total petroleum hydrocarbons, Modified Fenton, Oil sludge composting, Leachate

\section{A BSTRACT}

Article Info:

Received: 28 Jul 2020

Accepted: 09 Feb 2021

Available Online: 01 Aug 2021

Background and Aim The leachate from oil sludge compost into the environment, which is resistant to decomposition, causes several environmental problems. Therefore, it needs to be treated using efficient processes. This study aimed to investigate the efficiency of the modified Fenton process for treating these oil sludge leachates.

Methods \& Materials In this experimental study, leachate samples were collected, and then modified Fenton solution was prepared by adding $\mathrm{Fe}_{2}\left(\mathrm{SO}_{4}\right) 3$ to $\mathrm{H}_{2} \mathrm{O}_{2}$. Then the effect of time parameters $(15,30,60,90$, and 120 minutes), modified Fenton concentration of $20,50,100$, and $200 \mathrm{mg} / \mathrm{l}$ with pH 3, 5, 9, and three initial concentration of $\operatorname{COD}(500,1000$ and $1500 \mathrm{mg} / \mathrm{l})$ was examined.

Ethical Considerations This study has been registered in Arak University of Medical Sciences with codes 2645 and 2765 .

Results The reduction of COD and TPH in the optimal time of 60 minutes, initial COD of $500 \mathrm{mg} / \mathrm{l}, \mathrm{pH}$ of 3, and Fenton concentration of $200 \mathrm{mg} / \mathrm{l}$ were 9.04 and $77.42 \%$, respectively. The experiments showed that the removal efficiency of COD and TPH with increasing residence time and the concentration of modified Fenton are directly proportional and with decreasing, the initial concentration of $\mathrm{COD}$ and $\mathrm{pH}$ is inversely proportional. Conclusion The use of modified Fenton is an efficient method for treating leachate from petroleum sludge compost.

\section{Extended Abstract}

\section{Introduction}

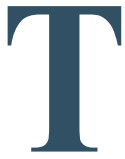

he discharge of oil sludge into the environment poses significant risks to human health and the environment [14]. Proper treatment can prevent the relevant contaminants $[5,6]$. One of the most critical treatment processes is biodegradation, such as com- posting [7], which produces contaminated leachate [8-10]. Koolivand et al. results $[11,12]$ showed that Hydrogen Peroxide and Fenton have good efficiency in reducing Total Petroleum Hydrocarbons (TPH). One of the advantages of the modified process is the ability to operate in a wide range of $\mathrm{pH}$ [13]. Some studies $[14,15]$ have been conducted to treat municipal waste leachate by Fenton. Other related studies have been done by Hosseini et al. [16], Attarian and Mokhtarani [17], Mahdad et al. [18], Hashemi et al. [19], and Mokhtarani et al. [20]. This study aimed to evaluate the

\section{* Corresponding Author:}

Ali Koolivand, PhD.

Address: Department of Environmental Health Engineering, Faculty of Health, Arak University of Medical Sciences, Arak, Iran.

Tel: +98 (86) 33686443

E-mail: akulivand@yahoo.com 
efficiency of the modified Fenton process in leachate treatment from petroleum sludge compost.

\section{Materials and Methods}

The leachate used in this study was obtained from the oil sludge composting process by the Windrow method. First, Fenton solution was prepared in the laboratory, and after preparing the desired concentrations, it was added directly to the reactors containing $200 \mathrm{ml}$ of compost leachate. In the Fenton solution, hydrogen peroxide to iron was set to about 10: 1 [21]. Iron was supplied by the chemical substance ferric sulfate $\mathrm{Fe}_{2}\left(\mathrm{SO}_{4}\right)_{3}$. Independent variables in this study included initial leachate concentration, modified Fenton concentration, reaction time, and $\mathrm{pH}$. The $\mathrm{pH}$ of the samples was adjusted at each stage using solutions of sulfuric acid and sodium at 3,5, and 9. After adding the desired concentrations $(20,50,100$, and $200 \mathrm{mg} / \mathrm{l})$ of modified Fenton solution to the test reactors, sampling was performed at 15, 30, 60, 90, and 120 minutes and COD and TPH of each sample were measured as dependent variables of the study. The initial COD of the models used in the experiments was about 500, 1000, and $1500 \mathrm{mg} / 1$. TNRCC 1005 and TNRCC 1006, provided by the Texas Department of Natural Resources, measured TPH [22].

\section{Results}

Table 1 shows the effect of $\mathrm{pH}$, initial leachate concentration, and the modified Fenton concentration on the COD reduction efficiency. Table 2 shows the effect of $\mathrm{pH}$ and the modified Fenton concentration on the TPH reduction efficiency of the leachate. The results showed the lower the $\mathrm{pH}$ of the leachate, the higher the COD removal efficiency. Therefore, naturally, the highest COD removal efficiency is obtained at $\mathrm{pH}=3$. At this $\mathrm{pH}$, the highest efficiency (more than $90 \%$ ) was obtained in 60 minutes with a concentration of $200 \mathrm{mg} / \mathrm{l}$ Fenton. According to the results, COD removal efficiency increased from 15 to 60 minutes, and no signifi- cant change in removal rate was observed for more than 60 minutes. Therefore, 60 minutes can be considered as the optimum reaction time of the modified Fenton. Also, it was found that the higher the concentration of modified Fenton, the higher the removal efficiency. Therefore, naturally, the highest COD reduction efficiency is related to the $200 \mathrm{mg} / \mathrm{l}$ modified Fenton concentration.

The results also showed that the higher the initial concentration of COD, the lower the removal efficiency, so the highest efficiency in COD was $500 \mathrm{mg} / \mathrm{l}$. According to Table 2, which shows the TPH reduction efficiency of the leachate sample in 60 minutes, at modified Fenton concentrations in the range of $20-200 \mathrm{mg} / \mathrm{l}$ and $\mathrm{pH}=3$, the TPH removal efficiency is about $31-77 \%$. Be. These cultivars are in the range of $25-72$ and $20-65 \%$ for $\mathrm{pH}=5$ and $\mathrm{pH}=9$, respectively.

\section{Discussion and Conclusion}

At a very low $\mathrm{pH}$, the formation of $\mathrm{Fe}\left(\mathrm{H}_{2} \mathrm{O}\right) 2+$, which reacts very slowly with hydrogen peroxide, reduces the number of hydroxyl radicals and thus the process efficiency. The decrease in efficiency in alkaline conditions is due to the conversion of $\mathrm{Fe}_{2}+$ to $\mathrm{Fe}(\mathrm{OH})_{3}$ precipitate, which causes decomposition of $\mathrm{H}_{2} \mathrm{O}_{2}$ and prevents the formation of $\mathrm{OH}$ radicals [23]. A similar study by Tengrui et al. [23] found an optimum $\mathrm{pH}$ of 3 that is consistent with the results of this study. The time required to complete the Fenton reaction depends on several factors, such as the concentration of Fenton used and the nature and concentration of the contaminant [23].

In Fenton's reaction, the removal efficiency does not change much from time to time. There may even be a slight decrease, which can be due to the production of some metabolites and intermediate products due to the decomposition of the intended contaminant [23]. The results of Farrokhi et al. [14] showed in the Fenton process, the highest amount of COD removal of waste leachate is obtained in

Table 1. COD reduction efficiency of leachate samples at different leachate concentrations at an optimum reaction time of 60 minutes

\begin{tabular}{cccccccc}
\hline $\begin{array}{c}\text { Modified Fenton Concen- } \\
\text { tration (mg/l) }\end{array}$ & $\mathbf{P H = 3}$ & $\mathbf{P H = 5}$ & $\mathbf{P H}=\mathbf{9}$ & $\mathbf{P H = 3}$ & $\mathbf{P H}=\mathbf{5}$ & $\mathbf{P H}=\mathbf{9}$ \\
\hline 20 & 36.77 & 32.25 & 29.38 & 19.00 & 16.59 & 15.00 \\
\hline 50 & 56.47 & 51.24 & 47.70 & 29.43 & 26.39 & 24.49 \\
100 & 89.63 & 89.30 & 81.09 & 48.01 & 45.75 & 41.66 \\
\hline 200 & 91.04 & 90.25 & 83.69 & 49.35 & $46 . .83$ & 43.18 \\
\hline
\end{tabular}


Table 2. Effect of modified $\mathrm{pH}$ and Fenton concentration on leachate TPH reduction efficiency at an optimum reaction time of 60 min

\begin{tabular}{|c|c|c|c|}
\hline \multirow{2}{*}{ Modified Fenton Concentration (mg/l) } & \multicolumn{3}{|c|}{$\mathrm{TPH}=170 \mathrm{mg} / \mathrm{l}$} \\
\hline & $\mathrm{PH}=3$ & $\mathrm{PH}=5$ & $\mathrm{PH}=9$ \\
\hline 20 & 31.27 & 25.89 & 20.20 \\
\hline 50 & 46.50 & 40.70 & 34.46 \\
\hline 100 & 73.66 & 68.24 & 60.67 \\
\hline 200 & 77.42 & 72.14 & 65.50 \\
\hline
\end{tabular}

the $\mathrm{pH}$ range of $3.5-3$, and the reaction time is 90 minutes, which is consistent with the results of this study. Among the important factors in the efficiency of the modified Fenton process are the concentration of Fenton used and the initial concentration of the contaminant [23].

The results of the oxidation study of Kerman city waste leachate using the Fenton process carried out by Malakoutian et al. [15] showed the maximum COD removal efficiency is obtained at a contact time of 75 minutes at $\mathrm{pH}=3$ iron concentration of $1400 \mathrm{mg} / \mathrm{l}$. Therefore, based on the results, it can be said that the use of low concentrations of modified Fenton has a slight effect on the removal of TPH, which is consistent with the results of Watts's study [24]. Overall, it can be concluded that the modified Fenton process can effectively reduce the COD of leachate from petroleum sludge compost.

\section{Ethical Considerations}

\section{Compliance with ethical guidelines}

This study has been registered in Arak University of Medical Sciences with codes 2645 and 2765.

\section{Funding}

Thihs study was supported by the Arak University of Medical Sciences.

\section{Authors' contributions}

All authors met the standard writing criteria based on the recommendations of the International Committee of Medical Journal Publishers.

\section{Conflicts of interest}

The authors declared no conflict of interest. 
بروسى كارايى فتثون اصلاحشده در تصفيه شيرابه حاصل از كميوست لجنهاى نفتى

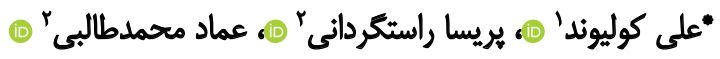

I. كروه مهيندسى بهداشت محيط، دانشكده بهداشت، دانشكاه علوميزشكى اراك، اراك، ايران.

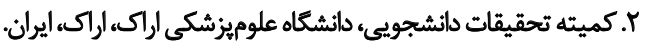

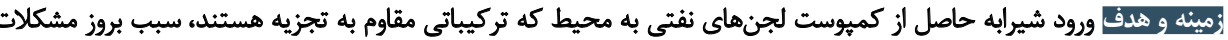

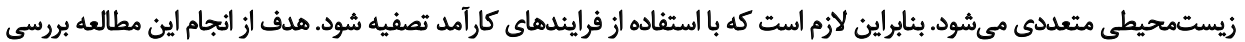

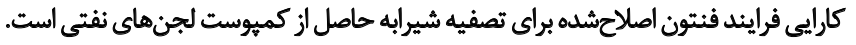

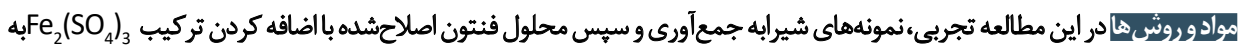

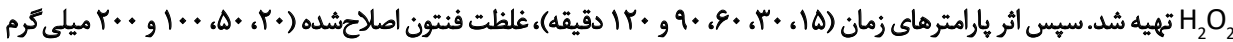

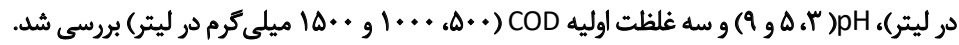

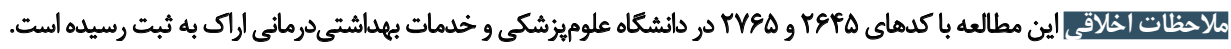

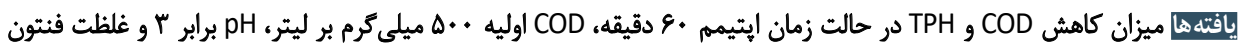

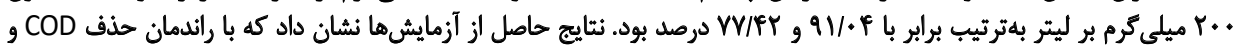

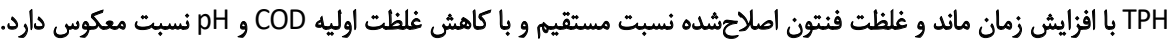

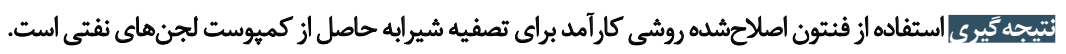

كليدوازوها:

كل هيدروكربنهاى كفتى، فنتون اصلاحشدهاه.

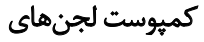
نفتي، شيرابه

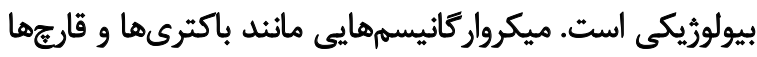

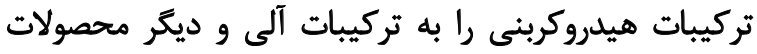

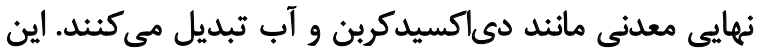

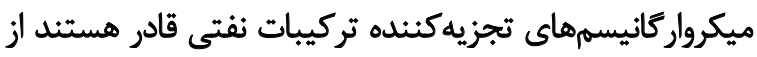

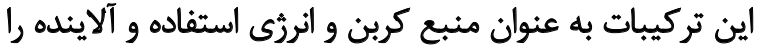

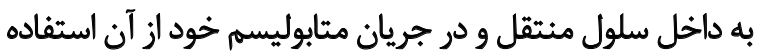

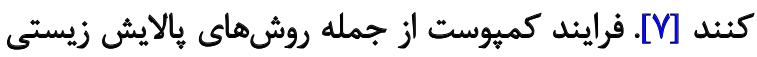

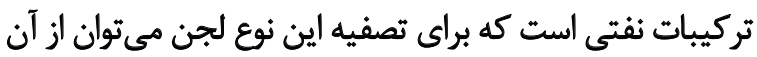

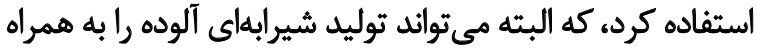

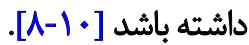

اكسيداسيون شيميايى يكى از روشهاي مؤثر تصفيه اين نوع

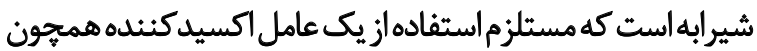

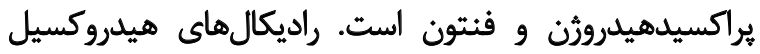
توليدى ناشى از واكنشهاى براكسيدهيدرورن و فنتون قادي اديد به

توسعه فعاليتهاى نفتى در كشورهاى نفتخيز سبب بروز

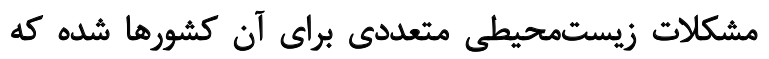

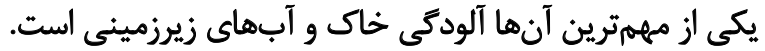

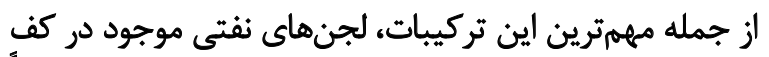

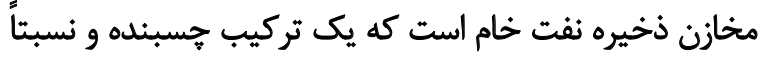

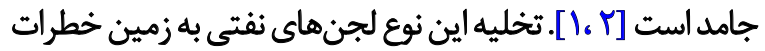

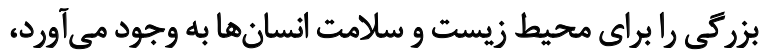

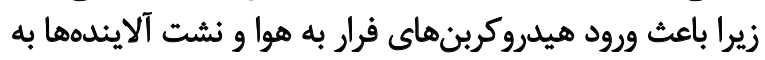

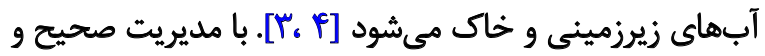

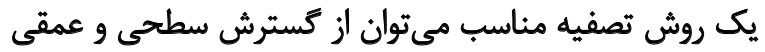

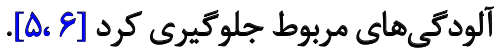

يكى از مهمترين فرايندها براى تصفيه تركيبات نفتى، تجزيه 
شد و در صورت كاهش با اضافه كردن آب، ميزان رطوبت تنظيم

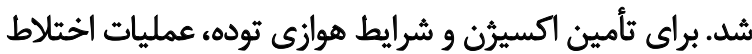

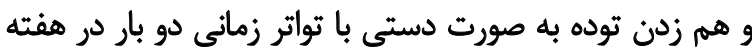

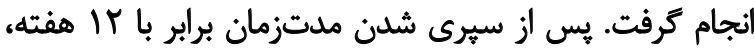

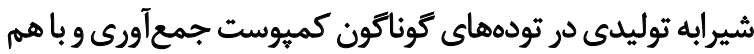

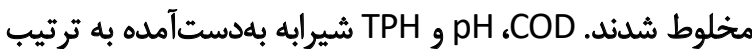

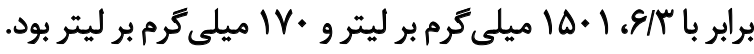

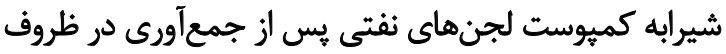

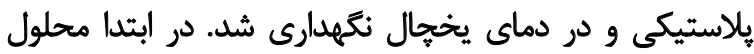

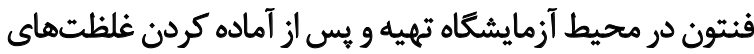

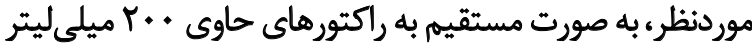

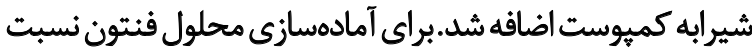

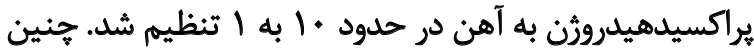

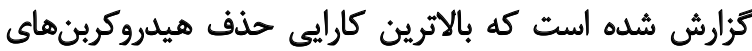

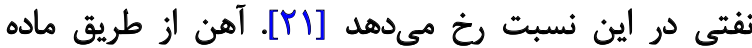
شيميايى سولفات فريك

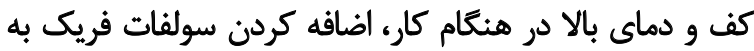

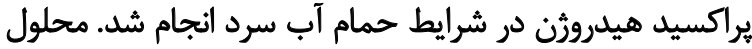

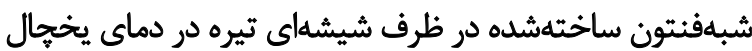

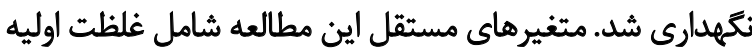

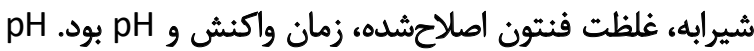

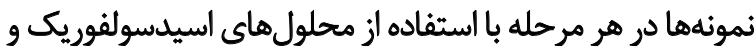

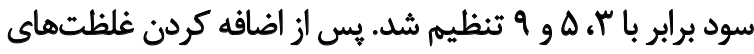

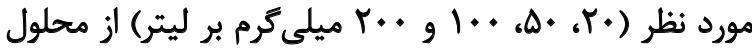

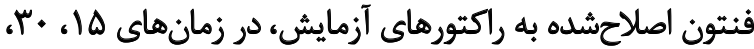

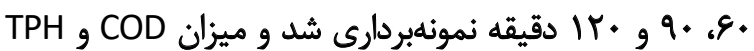

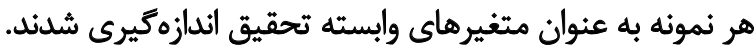

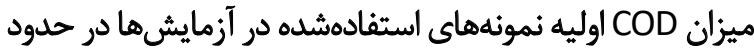

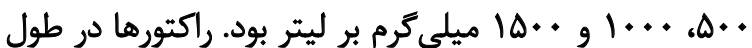

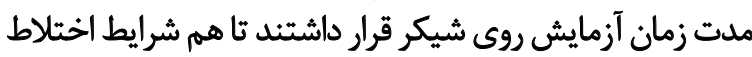

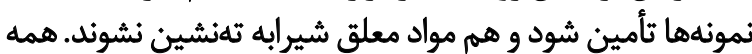
آزمايشها در دماي محيط و بادو باد بار تكرار انجام شد.

\section{TPH}

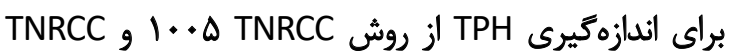

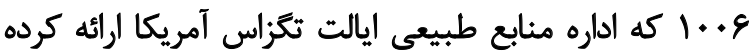

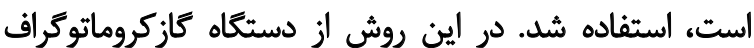

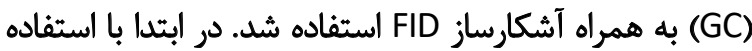

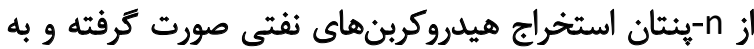
دنبال آن آشكارسازي با استفادهاز

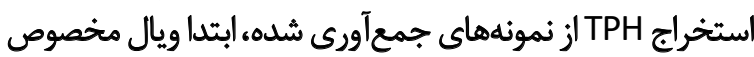

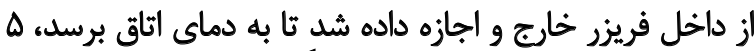

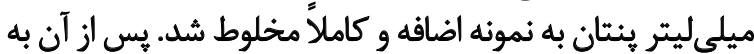

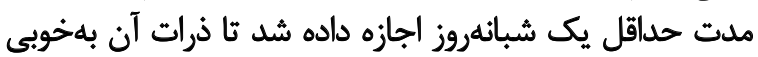

تجزيه و تخريب آلايندهاي آلى از جمله هيدركربنهاي نفتى

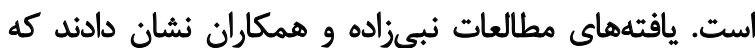

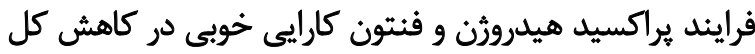

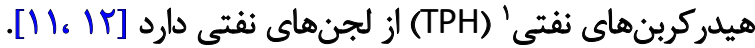

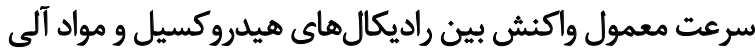

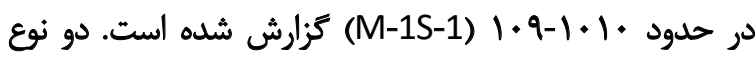

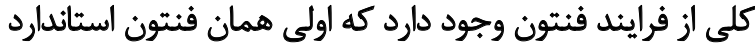

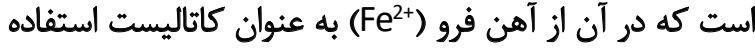

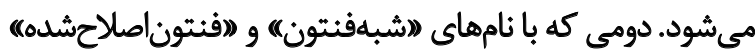

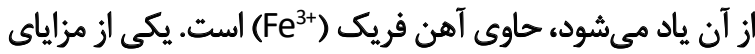

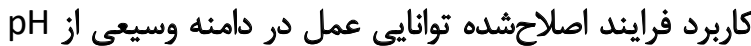

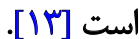

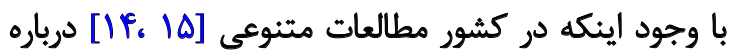

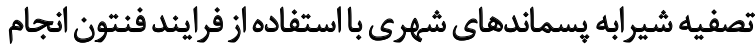

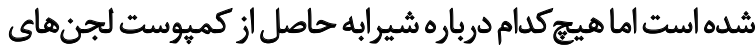

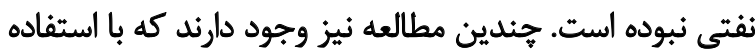

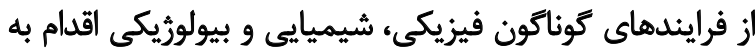

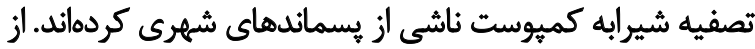

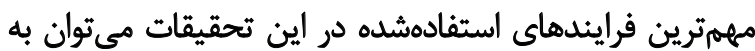

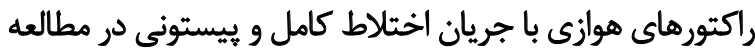

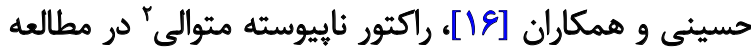

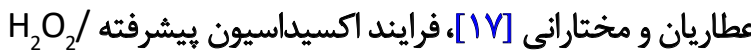

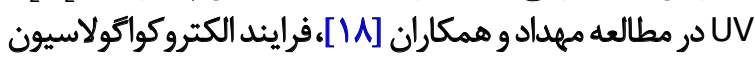

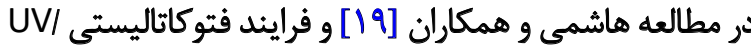
TiO

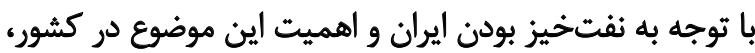

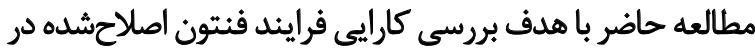
تصفيه شيرابه حاصل از كميوست لجن هارئ نفائ نفتى انجام شد.

$$
\text { مواد وروشها }
$$

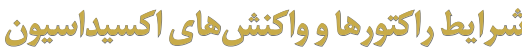

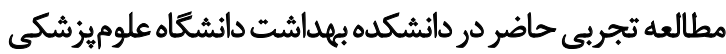

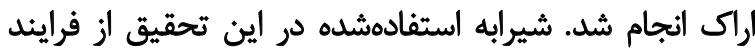

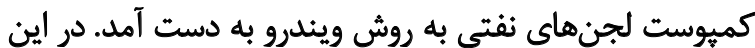

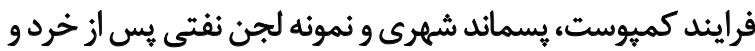

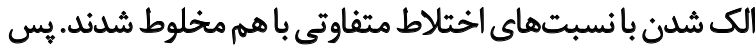
از اختلاط، نسبت C/N/P (توسط

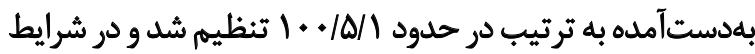

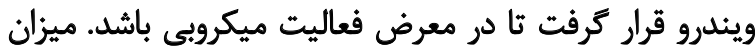

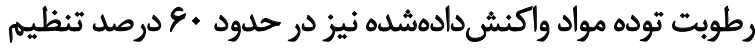

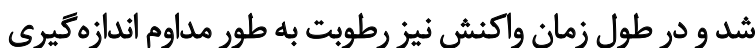

1. Total Petroleum Hydrocarbon

2. Styrene-butadiene rubber 


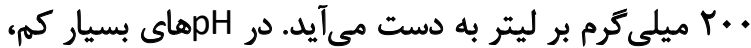

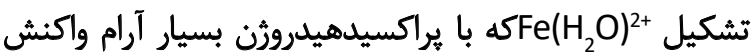

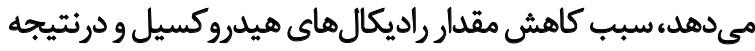

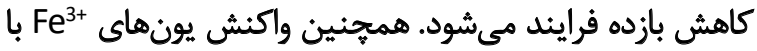

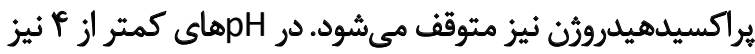

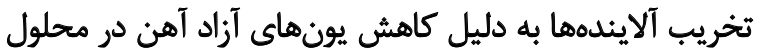

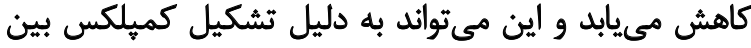

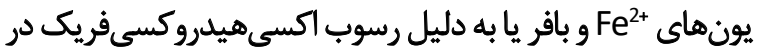

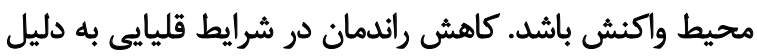

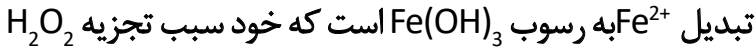

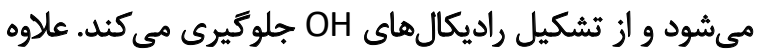

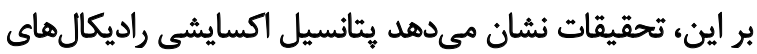

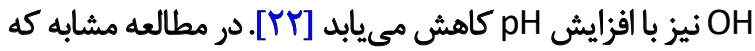

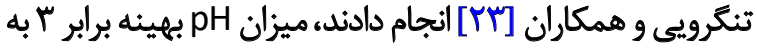

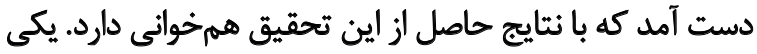

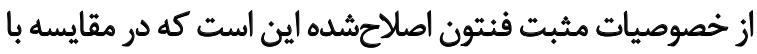

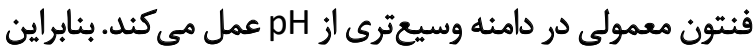

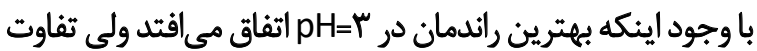

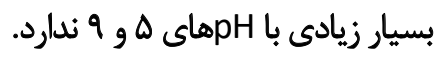

يكى ديكر از مهمثرين عوامل مؤثر بر راندمان فرايندهاى فئي

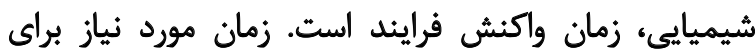

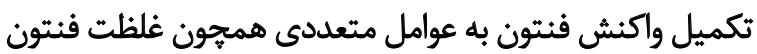

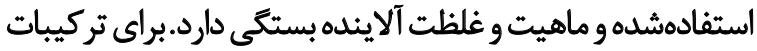

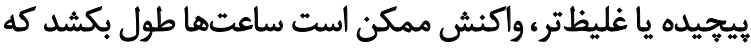

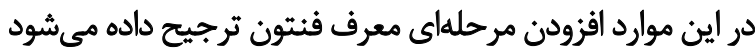

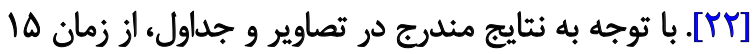

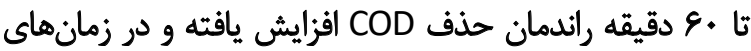
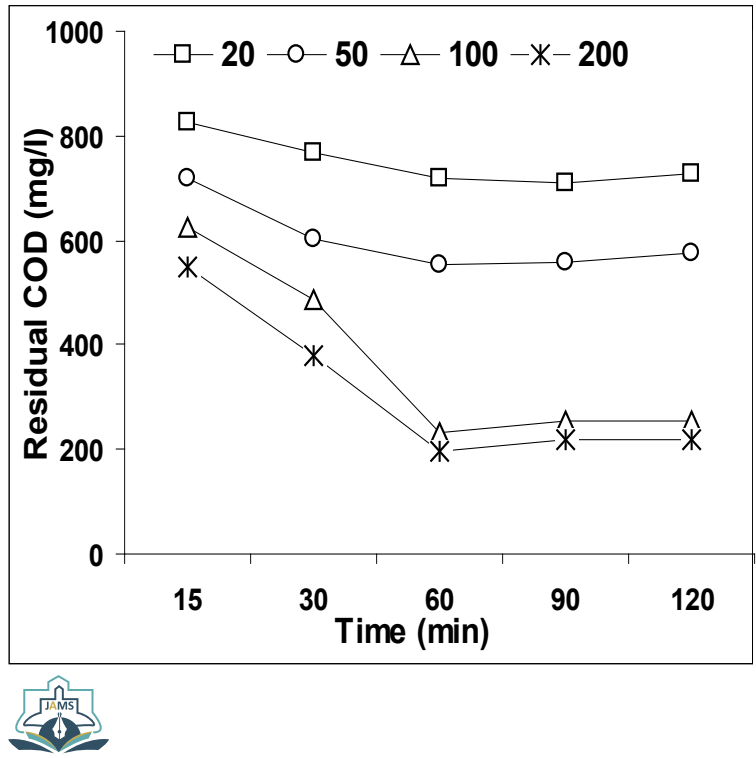

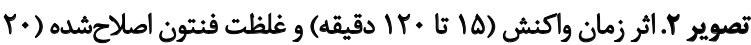

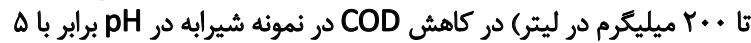

تهنشين شوند و درنهايت ميزان TPH آن به صورت مجموع كستره كربنها

\section{ووشئاي أماوى}

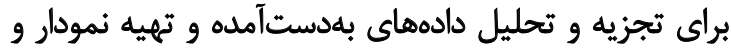

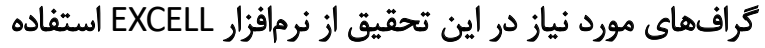

يافتهها

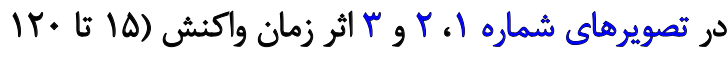

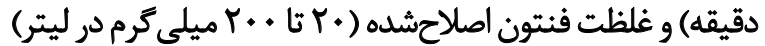

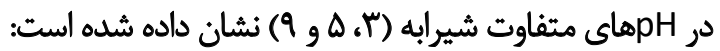
در جدول شماره ا اثر pH، غلظت اوليه شيرابه و همجنين

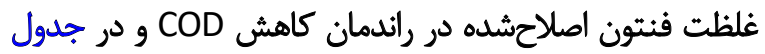

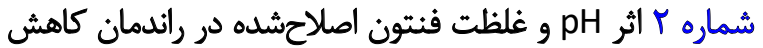

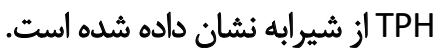

ب

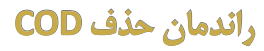

هحيط واكنش بر راندمان فرايند فنتون بسيار اثر كئار است،

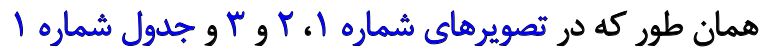

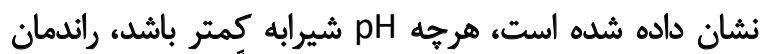

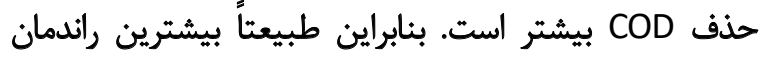

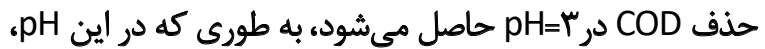

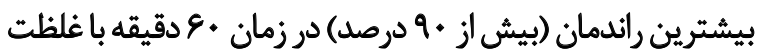
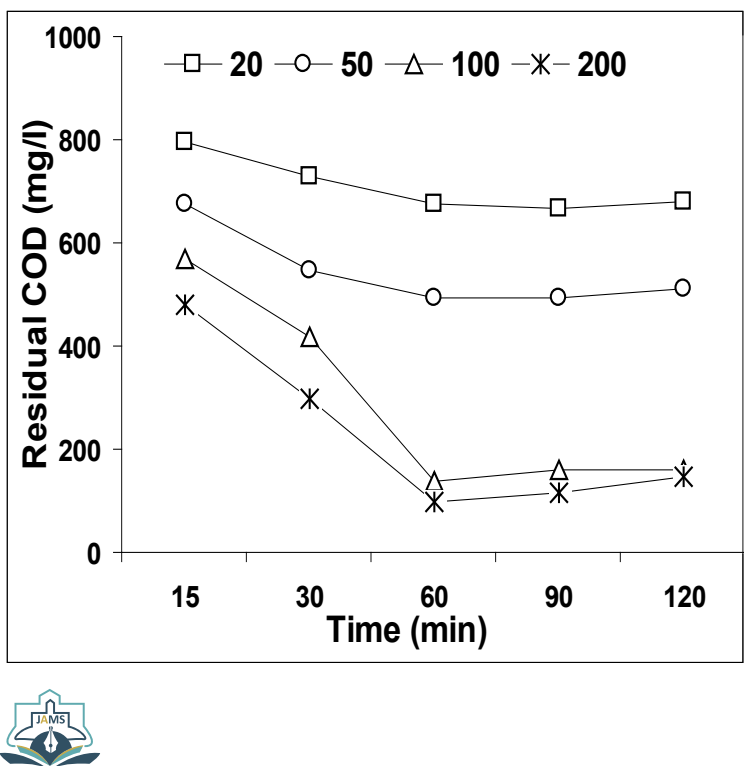

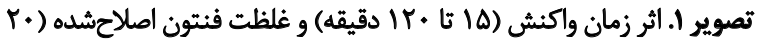

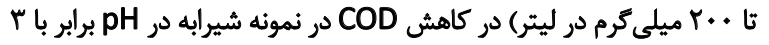




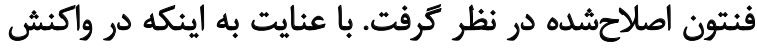

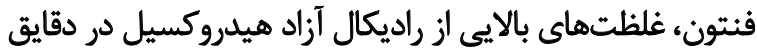

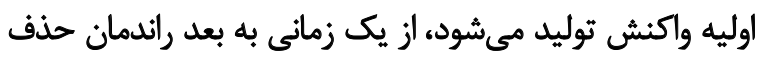

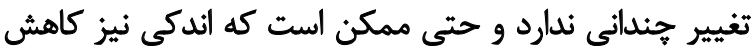

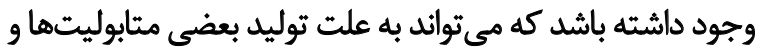

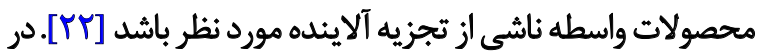

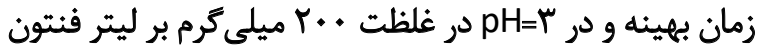

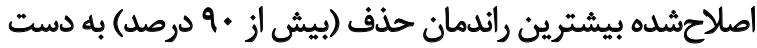

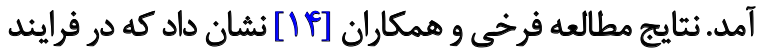

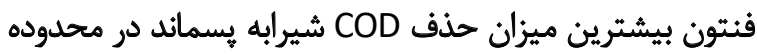

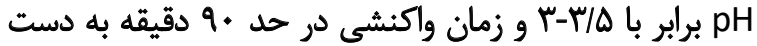
مى آيد كه با بتايج مطالعه حاضر همرخوانى دارد. از جمله فاكتورهاى مهم در راندمان فرايند فنتون اصلاحشده،

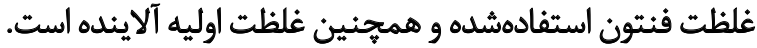

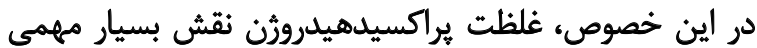

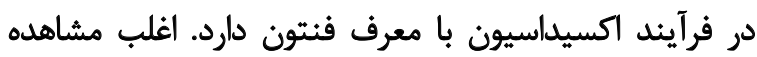

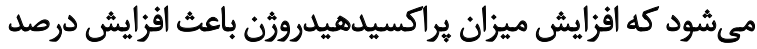

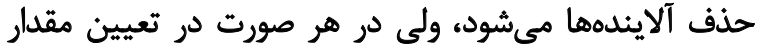

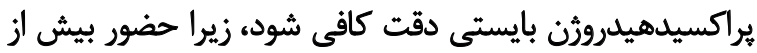

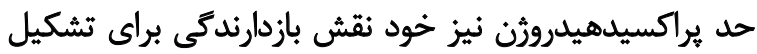
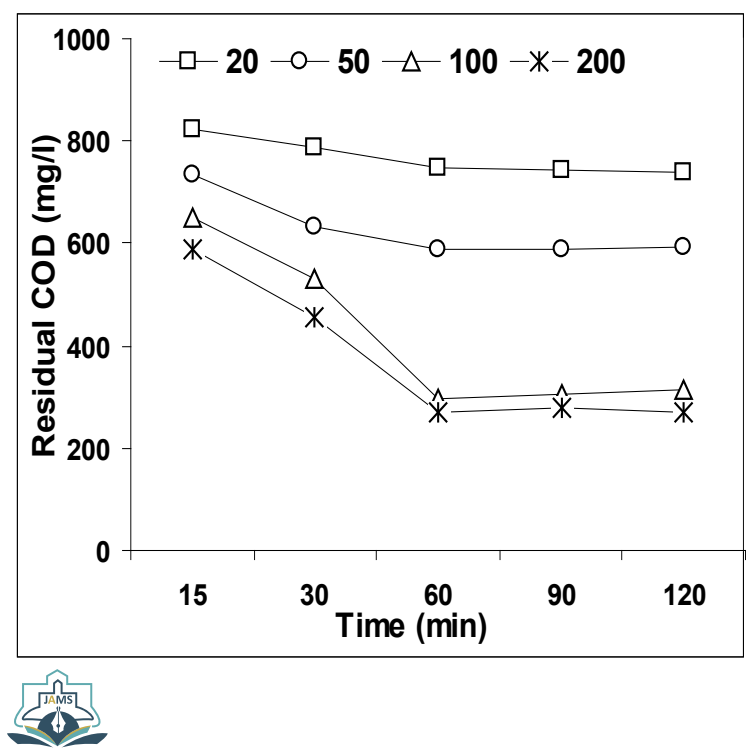

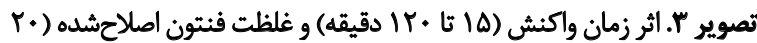

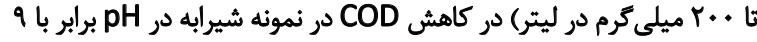
بيش از • ع دقيقه تغيير محسوسي در ميزان حذف مشاهده نشد.

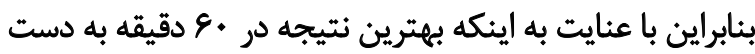

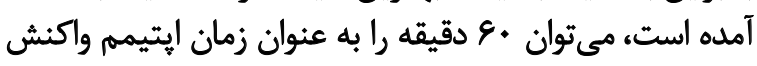

جدول ا. راندمان كاهش COD از نمونه شيرابه در غلظتهاى متفاوت شيرابه در زمان واكثش ايتيمهم •و دقيقه

\begin{tabular}{|c|c|c|c|c|c|c|}
\hline \multicolumn{3}{|c|}{$C O D=1500 \mathrm{mg} / \mathrm{l}$} & \multicolumn{3}{|c|}{$C O D=500 \mathrm{mg} / \mathrm{l}$} & \multirow{2}{*}{ غلظت فنتون اصلاحشده } \\
\hline $\mathrm{PH}=9$ & $\mathrm{PH}=5$ & $\mathrm{PH}=3$ & $\mathrm{PH}=9$ & $\mathrm{PH}=5$ & $\mathrm{PH}=3$ & \\
\hline $10 / \ldots$ & $18 / 09$ & $19 / \ldots$ & $r q / / 4$ & MT/TA & re/W & r. \\
\hline$r F / F q$ & rg/rq & rq/TH & $P V / V$. & $\Delta I / T H$ & $\Delta S / F V$ & $\Delta$. \\
\hline filfeg & $\Gamma \Delta / V \Delta$ & $P N=1$ & $A V / \cdot 9$ & $\Lambda q / \mathrm{r}$. & AV/R & $1 \ldots$ \\
\hline $\mathrm{NT/M}$ & fe/Ar & $F q / T \Delta$ & $\Delta r / E q$ & $q . / r \Delta$ & $91 / .7$ & r.. \\
\hline
\end{tabular}

جدول r. اثر pH و غلظت فنتون اصلاحشده در رائدمان كاهش TPH شيرابه در زمان واكنش ايتيمم •و دقيقه

\begin{tabular}{|c|c|c|c|}
\hline \multicolumn{3}{|c|}{$\mathrm{TPH}=170 \mathrm{mg} / \mathrm{l}$} & \multirow{2}{*}{ غلظت فُتتون اصلاحتشده (mg/l) } \\
\hline $\mathrm{PH}=9$ & $\mathrm{PH}=5$ & $\mathrm{PH}=3$ & \\
\hline$r+r$. & $r \Delta / A q$ & $r \mid / r y$ & r. \\
\hline melpes & $r+N$ & PE/Q. & $\Delta$. \\
\hline $8.18 \mathrm{~V}$ & ENTE & $V w / 88$ & 1.0 \\
\hline$\varepsilon_{\Delta / \Delta}$ & $n / / f^{*}$ & $W / R T$ & r.. \\
\hline
\end{tabular}




\section{ملاحظات اخلاقى \\ 亩}

اين مطالعه با كدهاي rFPA , rFED در دانشكاه علوميزشكى

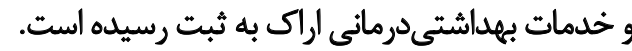

$$
\text { oth ob }
$$

ايـن يروهــش بـا حمايـت مالى دانشـعاه علوميزشـكى اراك اجرا شـد. أبرون

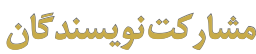

همه نويسندكان معيار هاى استاندارد نوشتن كميته بين المللى ناشران مجلات يزشكى را رعايت كردهائد.

cillia oble

بنابر اظهار نويسندكان اين مقاله تعارض منافع ندارد.
راديكال هاى هيدروكسيل دارد.از اين رو مقدار يراكسيدهيدروثن

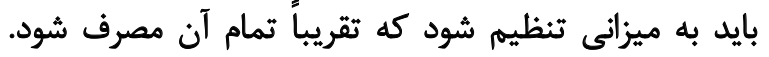

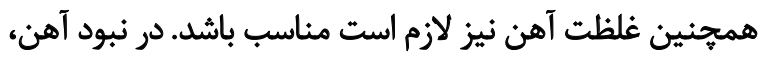

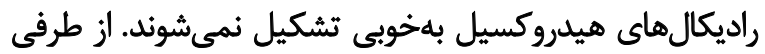

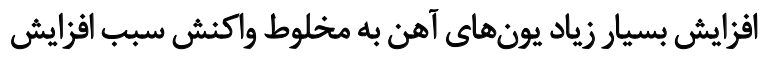

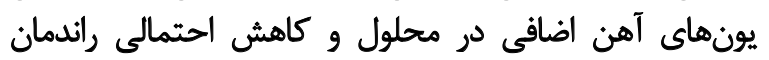

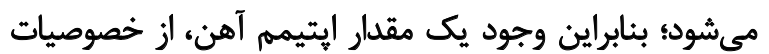

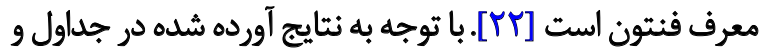

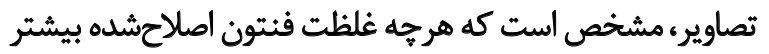

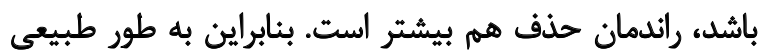

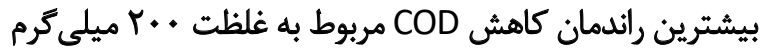

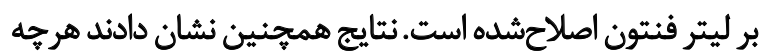

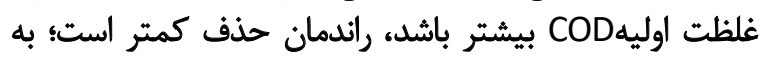

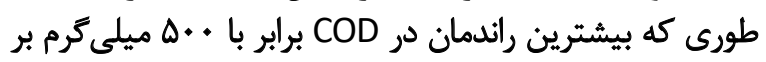

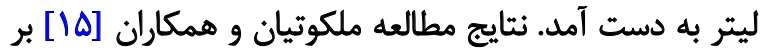

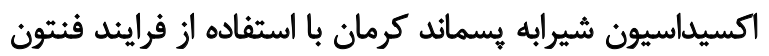

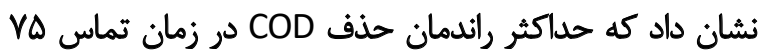

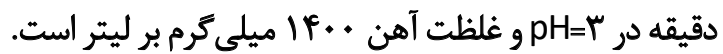

TPH أندمان هئف TPH

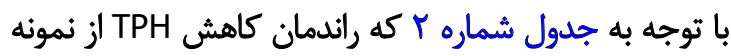

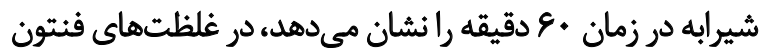

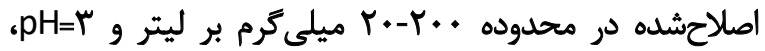

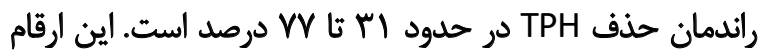

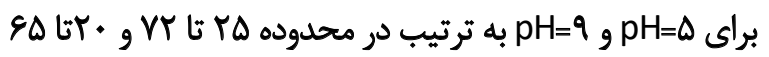

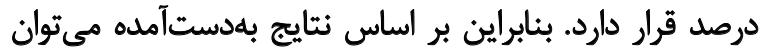

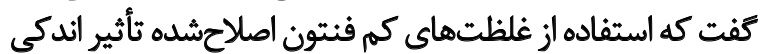

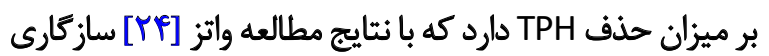

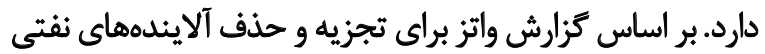

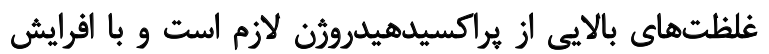

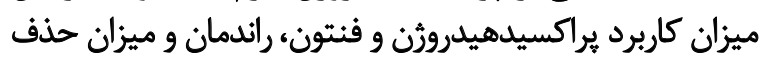
TPH

\section{نتيجلمَيرى}

فرايند فنتون اصلاحشده به شكل مؤثرى مي تواند COD شيرابه

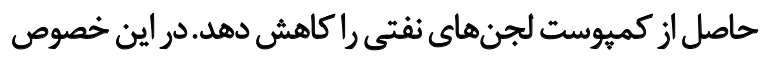

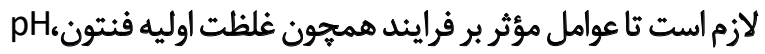

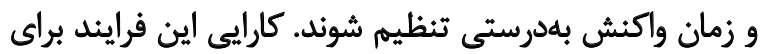

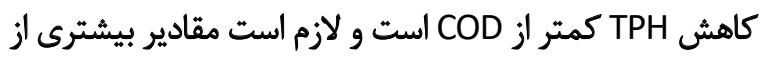
فنتون اصلاحشده استفاده شود. 


\section{References}

[1] Koolivand A, Naddafi K, Nabizadeh R, Saeedi R. Optimization of combined in-vessel composting process and chemical oxidation for remediation of bottom sludge of crude oil storage tanks. Environ Technol. 2018; 39(20):2597-603. [DOI:10.1080/09593330.2017.13 62037] [PMID]

[2] Varjani SJ. Microbial degradation of petroleum hydrocarbons. Bioresour Technol. 2017; 223:277-86. [DOI:10.1016/j.biortech.2016.10.037] [PMID]

[3] Thion C, Cébron A, Beguiristain T, Leyval C. PAH biotransformation and sorption by Fusarium solani and Arthrobacter oxydans isolated from a polluted soil in axenic cultures and mixed co-cultures. Int Biodeterior Biodegradation. 2012; 68:28-35. [DOI:10.1016/j. ibiod.2011.10.012]

[4] Zhang C, Qi J, Cao Y. Synergistic effect of yeast-bacterial co-culture on bioremediation of oil-contaminated soil. Bioremediat J. 2014; 18(2):136-46. [DOI:10.1080/10889868.2013.847402]

[5] Muangchinda C, Rungsihiranrut A, Prombutara P, Soonglerdsongpha $\mathrm{S}$, Pinyakong $\mathrm{O}$. $16 \mathrm{~S}$ metagenomic analysis reveals adaptability of a mixed-PAH-degrading consortium isolated from crude oil-contaminated seawater to changing environmental conditions. J Hazard Mater. 2018; 357:119-27. [DOI:10.1016/j.jhazmat.2018.05.062] [PMID]

[6] Zhang Y, Zhao Q, Jiang J, Wang K, Wei L, Ding J, et al. Acceleration of organic removal and electricity generation from dewatered oily sludge in a bioelectrochemical system by rhamnolipid addition. Bioresour Technol. 2017; 243:820-27. [DOI:10.1016/j.biortech.2017.07.038] [PMID]

[7] Poorsoleiman MS, Hosseini SA, Etminan A, Abtahi $\mathrm{H}$, Koolivand A. [The efficiency of acinetobacter radioresistens strain ka2 isolated from oily sludge for degrading of crude oil (Persian)]. J Arak Univ MedSci. 2019; 22(5):78-89. [DOI:10.32598/JAMS.22.5.78]

[8] Koolivand A, Godini K, Saeedi R, Abtahi H, Ghamari F. Oily sludge biodegradation using a new two-phase composting method: Kinetics studies and effect of aeration rate and mode. Process Biochem. 2019; 79:127-34. [DOI:10.1016/j.procbio.2018.12.003]

[9] Poorsoleiman MS, Hosseini SA, Etminan A, Abtahi H, Koolivand A. Bioremediation of Petroleum Hydrocarbons by using a two-step inoculation composting process scaled-up from a mineral-based medium: Effect of biostimulation of an indigenous bacterial strain. Waste and Biomass Valorization. 2021; 12(4):2089-96. [DOI:10.1007/ s12649-020-01140-z]

[10] Mnif I, Mnif S, Sahnoun R, Maktouf S, Ayedi Y, Ellouze-Chaabouni $\mathrm{S}$, et al. Biodegradation of diesel oil by a novel microbial consortium: Comparison between co-inoculation with biosurfactant-producing strain and exogenously added biosurfactants. Environ Sci Pollut Res Int. 2015; 22(19):14852-61. [DOI:10.1007/s11356-015-4488-5] [PMID]

[11] Koolivand A, Naddafi K, Nabizadeh R, Jafari AJ, Nasseri S, Yunesian $M$, et al. Application of hydrogen peroxide and fenton as pre-and post-treatment steps for composting of bottom sludge from crude oil storage tanks. Pet Scie Technol. 2014; 32(13):1562-8. [DOI:10.108 0/10916466.2012.697961]

[12] Koolivand A, Naddafi K, Nabizadeh R, Nasseri S, Jafari AJ, Yunesian $\mathrm{M}$, et al. Degradation of petroleum hydrocarbons from bottom sludge of crude oil storage tanks using in-vessel composting followed by oxidation with hydrogen peroxide and Fenton. J Mater Cycles Waste Manag. 2013; 15(3):321-7. [DOI:10.1007/s10163-013-0121-1]
[13] Nam K, Rodriguez W, Kukor JJ. Enhanced degradation of polycyclic aromatic hydrocarbons by biodegradation combined with a modified Fenton reaction. Chemosphere. 2001; 45(1):11-20. [DOI:10.1016/S0045-6535(01)00051-0]

[14] Farrokhi M, Kouti M, Mousavi G R, Takdastan A. [The study on biodegradability enhancement of landfill leachate by fenton oxidation (Persian)]. Iran J Health Environ. 2009; 2(2):114-23. https://www.sid. ir/en/journal/ViewPaper.aspx?id=160390

[15] Malakootian M, Ahmadian M, Loloei M. [Influence of fenton process on treatability of Kerman city solid waste leachate (Persian)] Iran J Health Environ. 2010; 3(2):123-34. http://ijhe.tums.ac.ir/article-1-118-en.html

[16] Hassani A, Mokhtarani N, Bayatfard A. [Post treatment of composting leachate using combination of aerobic completely mixed and plugs flow reactors (Persian)]. J EnvironSci Technol. 2012; 14(1):4-9 https://jest.srbiau.ac.ir/? action=articlelnfo\&article=2209\&lang=en

[17] Attarian P, Mokhtarani N. [Post-treatment of composting leachate by Sequencing Batch Reactor (SBR) (Persian)]. Modares Civil Eng J. 2018; 18(1):171-82. https://mcej.modares.ac.ir/article16-15839-en.html

[18] Mahdad F, Younesi H, Bahramifar N, Hadavifar M. [Optimization of compost leachate treatment using advanced oxidation process $\mathrm{h}_{2} \mathrm{O}_{2} / \mathrm{uv}$ (Persian)]. Modares Civil Eng J. 2017; 17(2):247-56. https:// mcej.modares.ac.ir/article-16-305-en.html

[19] Hashemi H, Alipor Samani E, Amin M M, Bina B. [Urvey on electrocoagulation process efficiency on Isfahan composting plant leachate treatment (Persian)]. J Health System Res. 2013; 9(9):969-78. http:// hsr.mui.ac.ir/article-1-669-en.html

[20] Mokhtarani N, Khodabakhshi S, Ayati B. [UV-TiO2 Photocatalytic degradation of compost leachate (Persian)]. Modares Civil Eng J. 2014 14(20):137-46. https://mcej.modares.ac.ir/article-16-951-en.html

[21] Goi A, Trapido M. Degradation of polycyclic aromatic hydrocarbons in soil: The Fenton reagent versus ozonation. Environ Technol. 2004; 25(2):155-64. [DOI:10.1080/09593330409355448] [PMID]

[22] Wu C, Chen W, Gu Z, Li Q. A review of the characteristics of Fenton and ozonation systems in landfill leachate treatment. Sci Total Environ. 2021; 762:143131. [DOI:10.1016/j.scitotenv.2020.143131] [PMID]

[23] Tengrui L, Al-Harbawi A, Jun Z, Bo LM. The effect and its influence factors of the Fenton process on the old landfill leachate. J Appl Sci. 2007; 7(5):724-7. [DOI:10.3923/jas.2007.724.727]

[24] Watts RJ. Hydrogen peroxide for physicochemically degrading petroleum-contaminated soils. Remediat J. 1992; 2(4):413-25. [DOI:10.1002/rem.3440020407] 
This Page Intentionally Left Blank 